\title{
A Low-Complexity Resource Allocation Algorithm for MIMO-OFDMA Multicast Systems with Spectrum-Guarantee Provisioning
}

\author{
Ioannis G. Fraimis and Stavros A. Kotsopoulos \\ Wireless Telecommunications Laboratory (WTL), Department of Electrical and Computer Engineering, University of Patras, \\ 26500 Rio, Greece \\ Correspondence should be addressed to Stavros A. Kotsopoulos, kotsop@ece.upatras.gr
}

Received 24 July 2011; Revised 4 November 2011; Accepted 27 November 2011

Academic Editor: Floriano De Rango

Copyright (C 2012 I. G. Fraimis and S. A. Kotsopoulos. This is an open access article distributed under the Creative Commons Attribution License, which permits unrestricted use, distribution, and reproduction in any medium, provided the original work is properly cited.

\begin{abstract}
We study the important problem of resource allocation for the downlink of Multiple-Input Multiple output (MIMO) Multicast Wireless Systems operating over frequency-selective channels and we propose a low-complexity but efficient resource allocation algorithm for MIMO-enabled OFDMA systems. The proposed solution guarantees a minimum spectrum share for each user while also takes advantage of the multicast transmission mode. The presence of multiple antennas in both transmitter and receiver offers spatial diversity to the system along with the frequency diversity added by the OFDMA access scheme. The computational complexity is reduced from exponential to linear and validation of the proposed solution is achieved through various simulation scenarios in comparison with other multicast and unicast reference schemes used in MIMO-OFDMA systems. Numerical results and complexity analysis demonstrate the feasibility of the proposed algorithm.
\end{abstract}

\section{Introduction}

Future wireless systems along with voice are envisioned to provide plethora of rich multimedia services like mobile TV, video conferencing, and so forth, with various bandwidth requirements [1-5]. The introduction of new applications such as streaming video and up-to-date information distribution services (e.g., news, stock market, weather forecasting, etc.) has brought about the need for communication between one sender and many receivers. Communication between one transmitter and multiple receivers can be achieved by either the unicast or the multicast transmission mode [1-8].

Works [1-4] study Multimedia Broadcast Multimedia Service (MBMS) delivery to large group of users. Particularly, in $[1,2]$ authors conclude that a hybrid unicast-multicast delivery offers the best system resource utilization, while in [3], the case of reserved resources for multicast services is investigated and in [4] the use of multiple transmit and receive antennas is considered for multicasting, in order to achieve higher data rates. However, the aforementioned studies refer to Wideband Code Division Multiple Access (WCDMA) mobile networks and Orthogonal Frequency Division Multiple Access (OFDMA) scheme is the modulation and multiple access scheme adopted for next-generation wireless systems [5-17].

OFDMA is based on Orthogonal Frequency Modulation (OFDM) scheme and helps exploit multiuser diversity in frequency-selective channels, since it is very likely that some subcarriers that are in deep fade for some users are in good channel state for at least one of the other users $[9,12,14]$. Because of its superior performance over frequency-selective channels, OFDMA is embedded in multicast technologies for the efficient transmission of multimedia streams to mobile devices using TV [5], and it is the preferred technology in the Broadband Wireless Access (BWA) standards [10]. Finally, authors in $[11,12]$ show that the optimal policy in order to eliminate intracell interference is the exclusive assignment of each subcarrier to only one user.

Dynamic resource allocation algorithms have been developed in order to exploit the multiuser diversity that OFDMA 


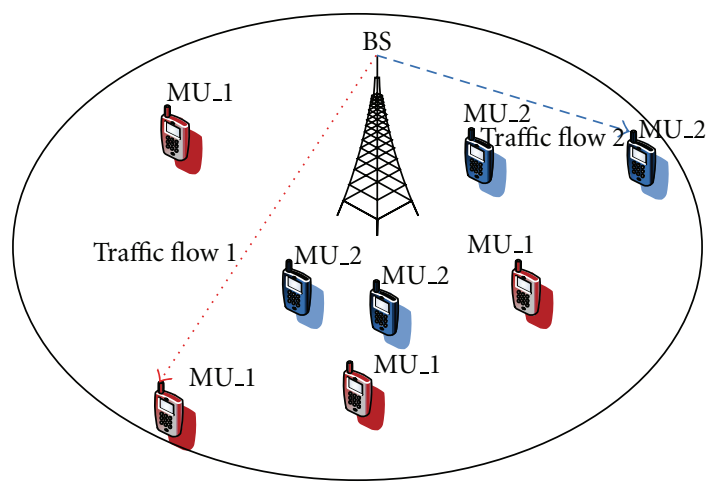

- Multicast group 1

- Multicast group 2

Figure 1: Multiple users demand the same data content.

offers and improve system capacity. More specifically, in [13] the available subcarriers are divided into a number of partitions and each user acts in parallel and attempts to select the partition with the highest average channel gain while in [14], capacity enhancement is achieved along with fairness criteria imposed to users. Similarly, authors in [15] introduced an iterative scheme so that quality-of-service (QoS) requirements of users are fulfilled with the objective to minimize the total transmit power.

On the other hand, when multiple users demand the same multimedia content (Figure 1), we can overcome the policy of exclusive subcarrier assignment to each user and allow many users to share the same subcarrier $[6,8]$. In this case, the transmitter establishes one link with a group of users which demand the same multimedia content. This kind of transmission is called multicast transmission in contrast to unicast transmission mode, wherein data has to be transmitted to each user separately. This is a great advantage of multicast mechanism which enhances significantly the system capacity.

To that end, authors in [6] propose a low-complexity algorithm which aims at improving the system data rate. The algorithm assigns each subcarrier to the group with the best channel conditions and the biggest number of member multicast users, and in [7] a heuristic algorithm for allocating resources is proposed in order to minimize power consumption. However, the aforementioned schemes focus on Single-Input Single-Output (SISO) systems and cannot be applied to MIMO systems directly [8], which are going to play a key role in future BWA communications $[18,19]$.

In fading environments, MIMO technology offers significant increase in link range and improvement in spectrum efficiency without additional spectrum and power requirements. Due to these properties, MIMO systems have received much attention by researchers and manufacturers. The block diagram of a MIMO system is given in Figure 2, wherein we can see the spatial diversity added to the system by the presence of multiple antennas. Spatial diversity in combination with the fading diversity of the OFDMA technology, can improve significantly the overall system performance.

The high computational complexity of optimally allocating subcarriers in MIMO systems [18] has driven many studies to propose exclusive allocation of each subcarrier to only one user despite the presence of multiple antennas in both transmitter and receiver [19-26]. In [17] we apply this approach, and more particularly we use the suboptimal subcarrier allocation criteria introduced in [19]. Similarly, authors in [8] apply a greedy suboptimal approach and allocate each subcarrier to the multicast group which offers the highest capacity gain without considering fair spectrum accessibility for users.

In this paper, motivated by works in $[6,8]$, we extend our work in [17] by addressing the resource allocation problem in an MIMO-aided wireless system, wherein multiple groups of users demand the same content with bandwidth access guarantees, contrary to schemes in $[6,8]$ which apply greedy policy and the only target is the maximization of the aggregate data rate. Moreover, the proposed scheme gives much better data rates compared to static Time Division Multiple Access (TDMA) scheme [27] and reduced complexity implementation.

The rest of the paper is organized as follows. Section 2 introduces the multiple antenna OFDMA multicast system model and formulates the optimization problem. The proposed suboptimal algorithm is analyzed in Section 3, while algorithm complexity issues are investigated in Section 4. Section 5 gives the simulation parameters and Section 6 presents the numerical results. Finally, Section 7 contains concluding remarks.

\section{System Model and Problem Formulation}

The block diagram of the considered MIMO-OFDM-based wireless multicast system is given in Figure 2, wherein the spatial multiplexing mode of MIMO is assumed as in $[8,17$, 19]. We consider a base station (BS) which serves $K$ wireless users over $N$ subcarriers. Let $N_{t}$ be the number of antennas at the BS and $N_{r}$ the number of antennas at each wireless user. Thus, the channel matrix of user $k$ on subcarrier $m$ is an $N_{r} \times N_{t}$ matrix denoted by

$$
\mathbf{H}_{k, n}=\left(\begin{array}{cccc}
h_{1,1}^{k, n} & h_{1,2}^{k, n} & \cdots & h_{1, N_{t}}^{k, n} \\
h_{2,1}^{k, n} & h_{2,2}^{k, n} & \cdots & h_{2, N_{t}}^{k, n} \\
\vdots & \vdots & \ddots & \vdots \\
h_{N_{r}, 1}^{k, n} & h_{N_{r}, 2}^{k, n} & \cdots & h_{N_{r}, N_{t}}^{k, n}
\end{array}\right),
$$

where $h_{r, t}^{k, n}$ is the channel gain from the $t$ th transmit antenna to the $r$ th receive antenna of user $k$ on the $m$ th subcarrier. It is also assumed that the eigenvalues of $\mathbf{H}_{k, n} \mathbf{H}_{k, n}^{T}$ are $\left\{\lambda_{k, m}^{(i)}\right\}_{i=1}^{M}$, where $M=\min \left(N_{r}, N_{t}\right)$. Hence, for certain values of $k$, $n$, a group of eigenchannels exists denoted by the above eigenvalues, according to SVD decomposition. Also, denoting the $N_{t} \times 1$ transmitted signal $\mathbf{X}_{k, n}=\left[x_{k, n}^{1}, x_{k, n}^{2}, \ldots, x_{k, n}^{N_{t}}\right]$ 


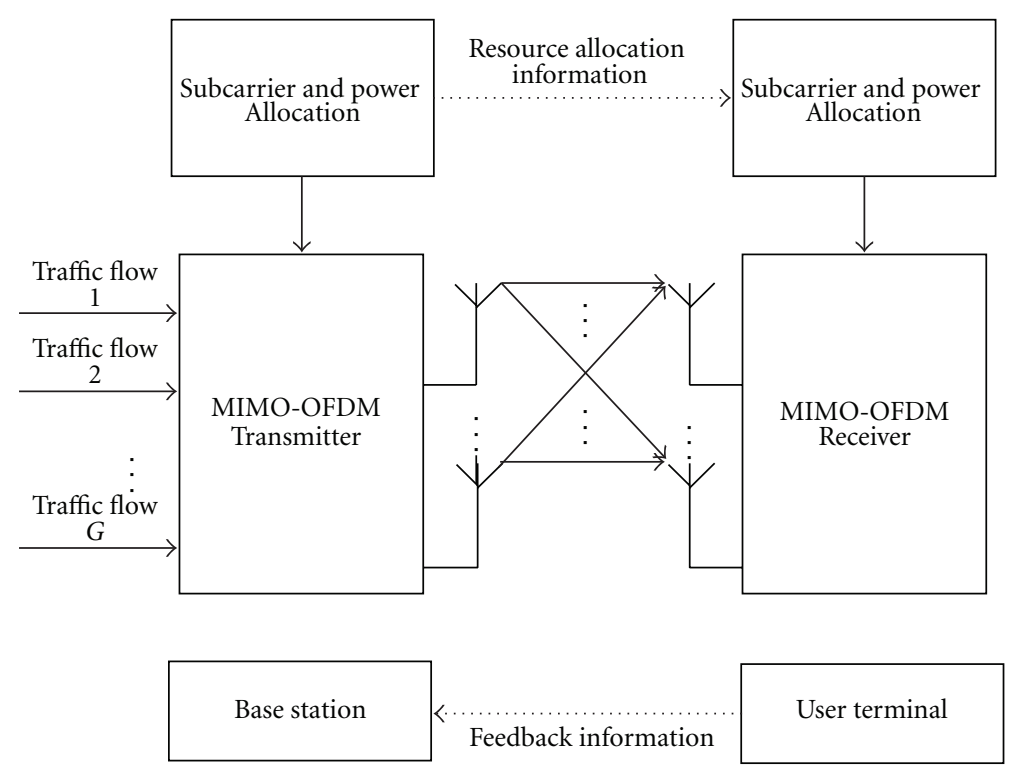

Figure 2: Block diagram of MIMO-OFDM multicast system.

and the $N_{r} \times 1$ received signal $\mathbf{Y}_{k, n}=\left[y_{k, n}^{1}, y_{k, n}^{2}, \ldots, y_{k, n}^{N_{r}}\right]$ is then

$$
\mathbf{Y}_{k, n}=\mathbf{H}_{k, n}\left(\sum_{k=1}^{K} \mathbf{X}_{k, n} p_{k, n}\right) \mathbf{N}_{k, n}
$$

where $\sum_{k=1}^{K} \mathbf{X}_{k, n} p_{k, n}$ is the overall transmitted signal on subcarrier $n$ and $p_{k, n}$ represents the power of subcarrier $n$ when assigned to user $k . \mathbf{N}_{k, n}$ is the $N_{r} \times 1$ noise vector.

Each user's bits are modulated into $N$ M-level Quadrature Amplitude Modulation (QAM) symbols, which are subsequently combined using the IFFT into an OFDMA symbol. For a square M-level QAM using Gray bit mapping, the Bit Error Rate (BER) can be approximated to within $1 \mathrm{~dB}$ for $r_{k, n}^{i} \geq 4$ and $\mathrm{BER}_{k, n}^{i} \leq 10^{-3}$ as in [28], being

$$
\begin{aligned}
\mathrm{BER}_{k, n}^{i} & \approx \frac{1}{5} \exp \left[\frac{-1.5 p_{k, n} \lambda_{k, n}^{i}}{N_{0}\left(2^{r_{k, n}^{i}}-1\right)}\right], \\
r_{k, n}^{i} & =\log _{2}\left(1+\frac{p_{k, n} \lambda_{k, n}^{i}}{N_{0} \Gamma_{k, n}^{i}}\right),
\end{aligned}
$$

where $\Gamma_{k, n}^{i}=-\ln \left(5 \mathrm{BER}_{k, n}^{i}\right) / 1.5$. For simplicity, it is assumed $\mathrm{BER}_{k, n}^{i}=\mathrm{BER}$ and $\Gamma_{k, n}^{i}=\Gamma$.

The great advantage of the multicast transmission is that data can be delivered to multiple receivers through a single transmission. However, each member user (MU) of a multicast group (MG) experiences a different Signal-toNoise Ratio (SNR) on a specific subcarrier from other users in the same group. In other words, achievable data rates by individual users in a group are not equal on a particular subcarrier, and a widely adopted approach is to transmit at the data rate determined by the MU with the worst channel condition in an MG $[6,8]$. This approach assures that a multicast service can be provided to all users within an MG.
Therefore, the BS transmits data to the $j$ th group through subcarrier $n$ with rate

$$
r_{j, n}=\arg \min _{k \in \mathcal{K}_{j}} \frac{W}{N} \sum_{i=1}^{M} \log _{2}\left(1+\frac{\lambda_{k, n}^{i} p_{k, n}}{N_{0} \Gamma}\right),
$$

where $\mathcal{K}_{j}$ denotes the user set of multicast group $j$ and $W$ refers to the total system bandwidth. Hence, the aggregate data rate of group $j$, with the help of (4), is

$$
R_{j, n}=\sum_{k \in K_{j}} r_{j, n}=\left|\mathcal{K}_{j}\right| \cdot r_{j, n},
$$

where $|\cdot|$ denotes the cardinality of the set.

In this paper, the following assumptions are used.

(i) The BS has perfect knowledge of channel state information (CSI) of all users in the system via dedicated feedback channels, and it is able to determine the maximum rate a user can receive data, as well as on which subcarrier the transmission takes place.

(ii) The transmitted signals experience slowly time varying fading, so the channel coefficients can be regarded as constants during the resource allocation process.

(iii) The transmission power is equally distributed among subcarriers; that is, $p_{k, n}=p_{n}=P_{t} / N, k=$ $1 \cdots K, n=1 \cdots N$. Water-filling power allocation brings marginal performance enhancement over fixed power allocation combined with Adaptive Coding and Modulation (ACM), as authors in [11] have proven.

(iv) We assume the subcarrier allocation matrix $X_{G \times N}=$ $\left[x_{j, n}\right]$, whereby $x_{j, n}=1$ if MG $j$ is assigned subcarrier $n$ and $x_{j, n}=0$ if not. A subcarrier can be shared by multiple users but only by users of the same MG. Each subcarrier can be assigned to only one MG. 
(v) $K$ wireless users are divided into $G$ MGs which demand $G$ different multicast services.

Considering the assumptions previous, the optimization problem in order to maximize the aggregate data rate while a minimum spectrum share is ensured for each $M G$ is formulated as follows:

$$
\max _{x_{j, n}, p_{j, n}} \sum_{j=1}^{G} \sum_{n=1}^{N} x_{j, n} R_{j, n}
$$

subject to

$$
\begin{gathered}
x_{j, n} \in\{0,1\}, \quad \forall j, n, \\
\sum_{j=1}^{G} x_{j, n}=1, \quad \forall n, \\
\sum_{n=1}^{N} x_{j, n} \geq C_{j}, \quad \forall j, \\
\sum_{j=1}^{G} C_{j} \leq N, \quad C_{j} \in Z_{+}, \\
\sum_{j=1}^{G} \sum_{n=1}^{N} x_{j, n} p_{j, n} \leq P_{t}, \\
p_{j, n} \geq 0, \quad \forall j, n .
\end{gathered}
$$

In the problem formulation, the binary variable $x_{j, n}$ represents the assignment of subcarrier $n$ to the multicast group $j$ and constrains (8) and (7) ensure that intracell interference is avoided since one subcarrier can only be allocated to at most one MG. Constraint (9) guarantees a portion of the available resources to each MG. The concept of minimum bandwidth assurance has also been studied in [16] for relay-enhanced systems, and it is also considered in our approach in [17] for MIMO systems, while (10) express that the subcarriers guaranteed for all groups cannot exceed the total number of available subcarriers in the system. Finally, constraints (11) and (12) express the BS power limitation, whereby $P_{t}$ denotes the total BS transmission power.

It is very hard to determine the optimal solution of the problem (6)-(12) within a designated time, since the problem in (6)-(12) is NP hard and has exponential complexity of $\mathcal{O}\left(G^{N}\right)$ (Section 4). It also involves both continuous and binary variables, and the nonlinear constraints increase the difficulty in finding the optimal solution. Hence, we resort to suboptimal heuristic algorithms which are more suitable for practical implementations.

\section{The Proposed Resource Allocation Algorithm}

This section provides and analyzes the proposed low complexity resource allocation (LCRA) algorithm. The pseudocode of the LCRA algorithm is given in Algorithm 1. For the sake of clarity, the pseudocode is divided into steps which are described as follows:
Inputs:

$$
\begin{aligned}
& \mathcal{N}=\{1,2, \ldots, N\}, \\
& \mathcal{G}=\{1,2, \ldots, G\}, \\
& \mathcal{C}=\left\{C_{1}, C_{2}, \ldots, C_{G}\right\} \text { such that }(10) \text { is fulfilled, } \\
& R_{G \times N}=\left[R_{j, n}\right] \% \text { computed by }(5) . \\
& \text { Outputs: } X_{G \times N}=\left[x_{j, n}\right] .
\end{aligned}
$$

(1) Initialization:

$$
x_{j, n}=0, \text { for } j=1 \cdots G \text { and } n=1 \cdots N .
$$

Inputs of the LCRA algorithm are the sets of the total available subcarriers $\mathcal{N}$, the set $\mathcal{G}$ of $\mathrm{MGs}$, the set $\mathcal{C}$ which represents the spectrum access guarantee of each $M G$, and the rate matrix $R_{G \times N}$ which denotes the attainable data rates of MGs on each one of the available subcarriers. Output is the subcarrier allocation matrix, and in step 1 subcarrier indicators $x_{j, n}$ are set to zero for all MGs and all subcarriers.

(2) While $\mathcal{C} \neq\{\}$ :

(i) Find $\tilde{j} \in g$ an $\tilde{n} \in \mathcal{N}$ with $R_{\tilde{j}, \tilde{n}} \geq R_{j, n} \forall j, n$

(ii) If $\sum_{n=1}^{N} x_{\tilde{j}, n} \geq C_{\tilde{j}} \%$ examines(9)
(a) $\mathcal{C} \leftarrow \mathcal{C} \backslash\left\{C_{\tilde{j}}\right\}$
(b) $g \leftarrow g \backslash\{\tilde{j}\}$

(iii) Else
(a) Set $x_{\tilde{j}, \tilde{n}}=1$
(b) $\mathcal{N} \leftarrow \mathcal{N} \backslash\{\tilde{n}\} \%$ satisfies (8).

In step 2, the BS seeks the matching of $\mathrm{MG} j^{*}$ and subcarrier $n^{*}$ which contributes the most to the total capacity. In the following, the algorithm examines if the selected MG has already its minimum spectrum portion (9). If that is true, the minimum spectrum index $C_{j *}$ the particular MG is excluded from the set $\mathcal{C}$ and consequently $\mathrm{MG} j^{*}$ is excluded from subsequent iterations of this part of the LCRA algorithm. In case the MG has not been allocated its minimum number of channels, the subcarrier $n^{*}$ is allocated to MG $j^{*}$ and then the subcarrier $n^{*}$ is excluded from the set of available subcarriers $\mathcal{N}$ in order to fulfill (8). The procedure is repeated until the set $\mathcal{C}$ is empty or, in other words, each MG has got its minimum subcarrier allotment. The procedure could also stop if there were no more available subcarriers, despite the fact that some MGs may have lower number of subcarriers than $C_{j}, j=1 \cdots G$. However, this situation is excluded because of (10).

(3) While $\mathcal{N} \neq\{\}$ :

(i) Find $j^{*}, n^{*}$ such that $R_{j^{*}, n^{*}} \geq R_{j, n} \forall j, n$

(ii) Set $x_{j^{*}, n^{*}}=1, \mathcal{N} \leftarrow \mathcal{N} \backslash\left\{n^{*}\right\} \%$ satisfies(8)

(iii) Update (6).

In step 3, if unallocated subcarriers exist, those are allocated according to the criterion of maximizing the aggregate data rate by allocating a subcarrier to the group with the best channel condition among all MGs. Then the selected subcarrier is excluded from the set $\mathcal{N}$ (satisfaction of (8)). This part enhances the system capacity. 


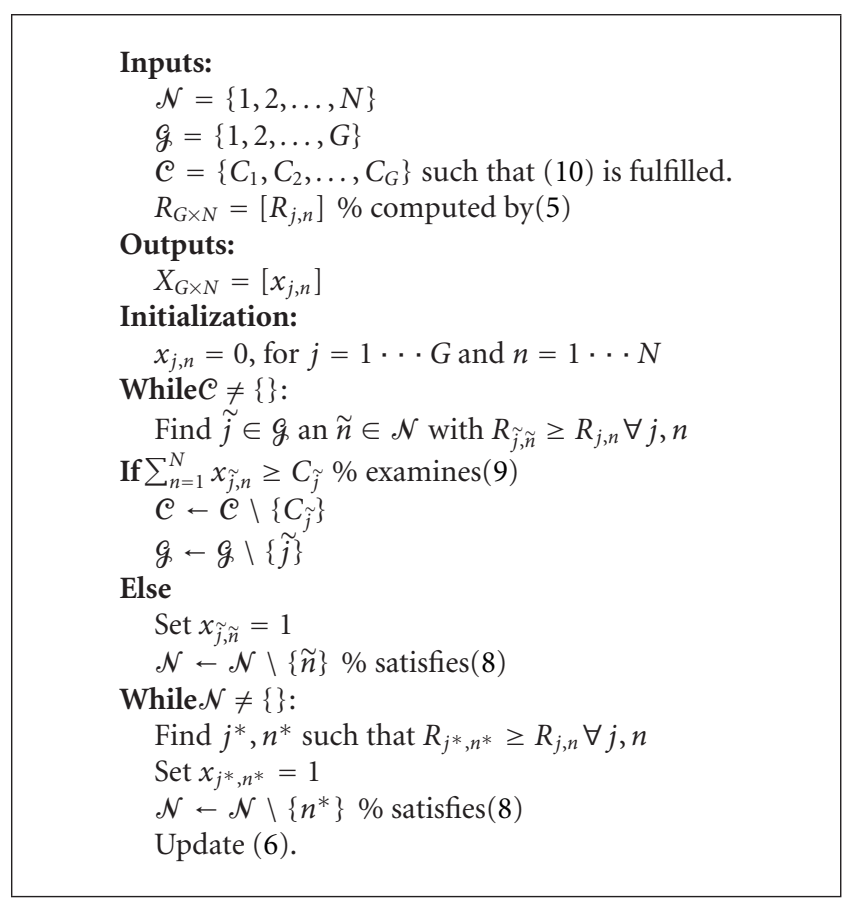

Algorithm 1:

TABLE 1: Algorithm complexity.

\begin{tabular}{lcccc}
\hline Exhaustive search & LCRA & {$[17]$} & Optimal user selection [18] & Subcarrier allocation in [8] \\
\hline $\mathcal{O}\left(G^{N}\right)$ & $\mathcal{O}(G \times N)$ & $\mathcal{O}(K \times N)$ & $\mathcal{O}\left(K^{\left\lfloor N_{T} / N_{R}\right\rfloor N}\right)$ & $\mathcal{O}(G \times N)$ \\
\hline
\end{tabular}

\section{Complexity Analysis}

The problem described by (6)-(12) is a binary integer programming problem with nonlinear constraints and finding the optimal solution requires $G^{N}$ possible subcarrier assignments. As a result the exhaustive search has an exponential complexity $\mathcal{O}\left(G^{N}\right)$ with respect to the number of subcarriers.

In step 1, the algorithm requires constant time in order to form the involved sets and the power portion for each subcarrier.

In the second step, the pair of group and subcarrier which gives the highest $R_{j, n}$ among $G$ MGs is found. In case an MG has been assigned its minimum channel portion, it is excluded from subsequent operations. In the worst case $G$ comparisons are required for each one of the available subcarriers. For $N$ subcarriers, we need $G \times N$ operations. Therefore, the complexity of this step reaches $\mathcal{O}(G \times N)$.

Step 3 searches for the best MG $j^{*}$ among $G$ MGs for the remaining $N-\sum_{j=1}^{G} C_{j}$ unallocated subcarriers. This step demands $G \times\left(N-\sum_{j=1}^{G} C_{j}\right)$ comparisons at most and therefore the $\mathcal{O}\left(G \times\left(N-\sum_{j=1}^{G} C_{j}\right)\right)$ complexity.

The overall LCRA complexity is upper bounded by $\mathcal{O}(G \times N)$ order of complexity, whereby the complexity of [17] is $\mathcal{O}(K \times N)$ and always $G \leq K$. The computational complexity of the LCRA has a form of $\mathcal{O}\left(L^{\delta}\right)$, with $L=G \times N$ and $\delta=1$ which is a linear [29], and the proposed LCRA scheme is a linear-time algorithm which is very efficient compared to the number of operations $G^{N}$ for the exhaustive search.

It is worth mentioning that the design assumptions that only users of the same MG are capable of sharing a subcarrier and the spatial multiplexing MIMO mode we adopted, reduce significantly the system complexity. Exhaustive search for the optimal user selection for MIMO systems requires $\mathcal{O}\left(K^{\left\lfloor N_{T} / N_{R}\right\rfloor N}\right)$, assuming that, for every subcarrier, we select a subset of users and every selected user will use the full dimension of its receive signal space. Hence, finding optimal solution is prohibitive even for moderate values of $K$ and $N$ [18]. In our problem formulation, the complexity is also independent of the number of receive and transmit antennas and this is a feature of great importance since the computation complexity of MIMO-OFDMA systems increases significantly by the presence of multiple antennas [18]. Table 1 summarizes computational complexities of the proposed LCRA algorithm and other reference schemes.

\section{Simulation Models and Parameters}

We consider an OFDMA system with $N=128$ subcarriers, wherein a BS serves $G$ MGs and each one has equal number of users. Both BS and users are equipped with multiple antennas, and we consider $N_{r}=N_{t}=2$ for our simulations. The available spectrum is $W=1 \mathrm{MHz}$ and the Additive White Gaussian Noise (AWGN) is $N_{0}=-80 \mathrm{dBW} / \mathrm{Hz}$ 
TABLE 2: Simulation parameters.

\begin{tabular}{lc}
\hline Parameters & Values \\
\hline Bandwidth & $1 \mathrm{MHz}$ \\
Number of subcarriers & 128 \\
Number of transmit antennas & 2 \\
Number of receive antennas & 2 \\
Fast fading & Jakes Model \\
Number of multipath components (taps) & 6 \\
AWGN spectral density (single-sided) & $-80 \mathrm{dBW} / \mathrm{Hz}$ \\
Number of users & 16 \\
Maximum doppler shift & $30 \mathrm{~Hz}$ \\
\hline
\end{tabular}

(single-sided PSD). In all simulations presented in this section, we consider frequency-selective channel which consists of six independent Rayleigh multipath components (taps) for every downlink transmission path between any of the $N_{t}$ transmit antennas and $N_{r}$ receive antennas of each multicast user. As in $[9,14,30]$, an exponentially decaying power profile is considered, whereby the ratio of the energy of the $l$ th tap to the first tap is equal to $e^{-2 l}$. We also assume a maximum delay spread of $5 \mu \mathrm{s}$ and maximum doppler of $30 \mathrm{~Hz}$. In all simulation scenarios the number of channel realizations is equal to 1000 and 10 time samples for each realization are used. The main simulation parameters are summarized in Table 2.

In all scenarios, the proposed LCRA algorithm is compared for different values of $C_{j}$, with the subcarrier allocation method proposed in [8] and static TDMA allocation proposed in [27] for multiple MGs. According to TDMA algorithm, each user is given a fair share of the channel resource regardless of the channel state. In our case, we apply the TDMA methodology for each MG according to (4) and (5). We find the user that determines the data rate of each group, otherwise the user with worst channel conditions on a subcarrier and we apply TDMA based on each MG. In order to distinguish the TDMA applied in multicast mode and unicast mode, we denote TDMA-MC the multicast TDMA algorithm and TDMA-UC the unicast TDMA algorithm. Both in the algorithm in [8] and in static TDMA algorithm, uniform power distribution is used. In addition, in our simulations we include popular unicast schemes like max-SNR scheduler and unicast schedulers with minimum bandwidth assurance for each user. For the unicast scheme with minimum bandwidth assurance, we use our proposed algorithm in [17], which fits for MIMO systems. For max-SNR scheduler, fairness is not a design priority and assigns any subcarrier to the user with the highest SNR. Finally, in all simulations, different variants of the LCRA scheme are considered.

Each variant is determined by the value of $C_{j}$ which is equal for all MGs in each variant. In the simulations scenarios described in the next section, for each variant $C_{j}=$ $\lfloor N /(m \cdot G)\rfloor$, where $m \in Z_{+}$. In case of unicast transmissions, $m$ is substituted by $w$. The relationship between these two variables for users in each MG is $w=K_{j} \cdot m, j=1, \ldots G$.
Hence, for unicast transmissions, there is $c_{k}=\lfloor N /(w \cdot G)\rfloor$ and we can see that as $m$ increases, the number of guaranteed resources for each MG decreases.

It is important to highlight that as the number of MUs in an MG tends to infinity, the ergodic system capacity becomes independent of the MG size [31].

We validate the proposed scheme based on: (1) The spectral efficiency and (2) the fairness pointer. Spectral efficiency is defined by (13). $S_{E}$ is the total system data rate averaged by the system bandwidth:

$$
S_{E}=\frac{\sum_{j=1}^{G} \sum_{n=1}^{N} x_{j, n} R_{j, n}}{W},
$$

Fairness pointer is given in (14), where $F_{p}$ is in the range of 0 and 1 . If resources are equally partitioned to all MGs, then the pointer achieves $1[14,30]$ :

$$
F=\frac{\left(\sum_{j=1}^{G} \sum_{n=1}^{N} R_{j, n}\right)^{2}}{G \cdot \sum_{j=1}^{G}\left(\sum_{n=1}^{N} R_{j, n}\right)^{2}} .
$$

In case of unicast transmission, (14) is formed as follows:

$$
\mathcal{F}=\frac{\left(\sum_{k=1}^{K} \sum_{n=1}^{N} b_{k, n}\right)^{2}}{K \cdot \sum_{k=1}^{K}\left(\sum_{n=1}^{N} b_{k, n}\right)^{2}},
$$

where, $b_{k, n}$ denotes the achieved bit rate of user $k$ on subcarrier $n$.

\section{Numerical Results}

In this section, we give the performance of the LCRA in comparison with the reference multicast and unicast schemes described in the previous section. We launch simulations for various scenarios and those are outlined in the following.

6.1. LCRA Scheme versus SNR. In Figures 3 and 4, we have set target BER $=10^{-6}$ and the SNR varies from 0 to $40 \mathrm{in}$ increment of 5 . The number of multicast groups is $G=4$ for with $\left|\mathcal{K}_{1}\right|=\left|\mathcal{K}_{2}\right|=\left|\mathcal{K}_{3}\right|=\left|\mathcal{K}_{4}\right|=4$ each one. Figure 3 shows the total system data rate versus the average SNR for the proposed LCRA scheme in comparison with the reference multicast and unicast schemes described in the beginning of the section, while Figure 4 gives the fairness pointer against the average SNR.

From Figure 3, we see that multicast transmissions enjoy higher spectral efficiency than the reference unicast schemes and all LCRA variants achieve lower spectral efficiency than the algorithm in [8], wherein fairness is not considered. On the other hand, in Figure 4 LCRA variants show more fairness than the algorithm in [8] which is an objective goal of the proposed approach.

6.2. Proposed LCRA Algorithm versus BER. This simulation scenario investigates spectral efficiency and fairness of the LCRA algorithm along with the other reference schemes against the average BER. Average BER varies from $10^{-7}$ 


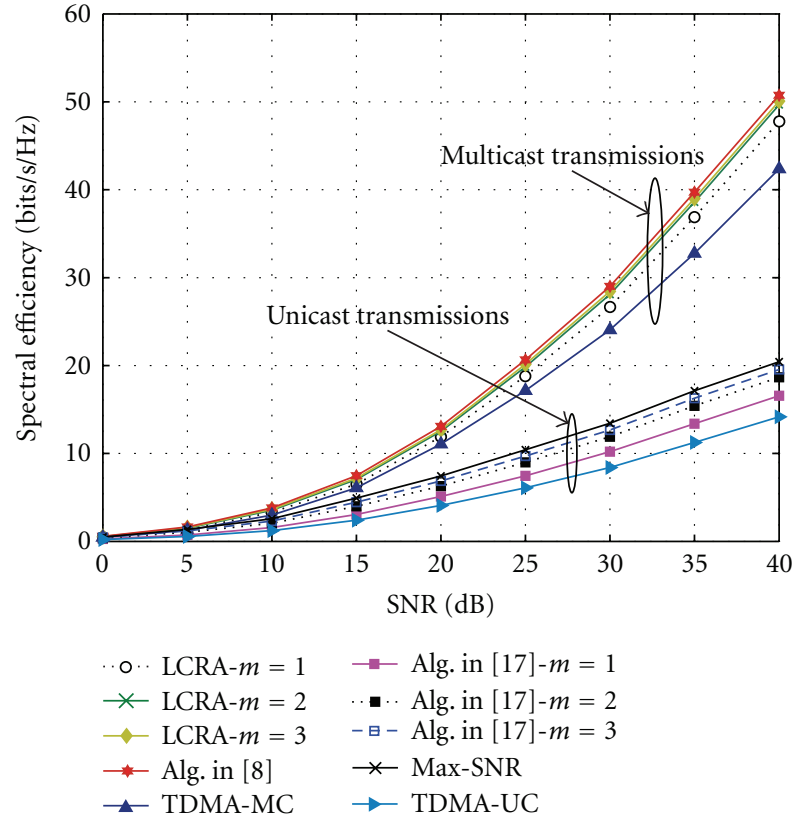

FIGURE 3: System data rate versus average SNR.

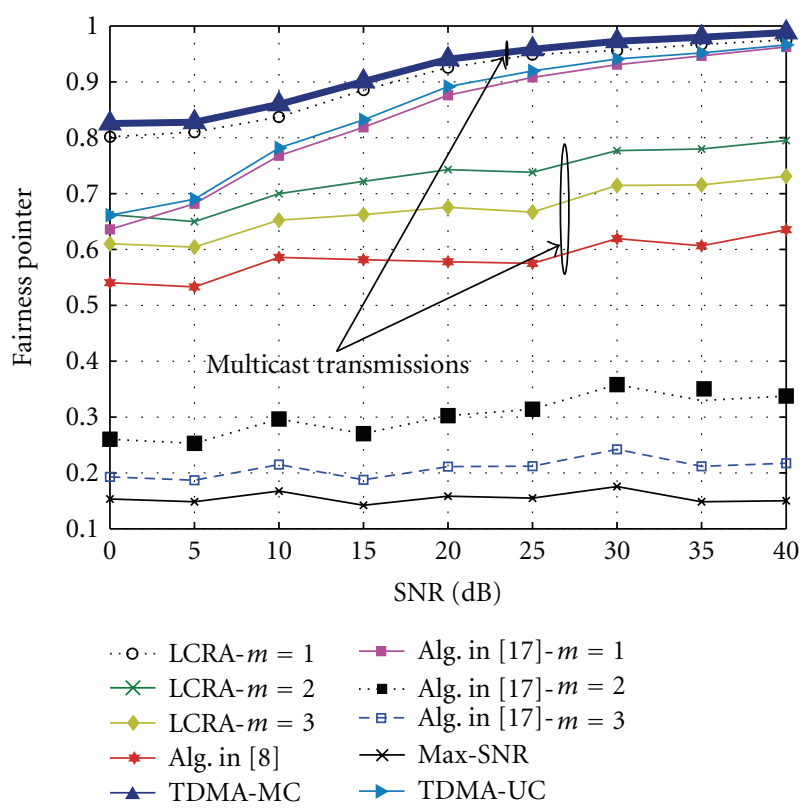

FIgURE 4: Fairness versus average SNR.

to $10^{-3}$ with average SNR being set to $20 \mathrm{~dB}$. Figure 5 plots the spectral efficiency for various values of BER and shows that as the average BER grows the same happens with the achieved spectral efficiency. However, multicast schemes provide superior performance over unicast schemes. Specifically, the algorithm in [8] and max-SNR scheme give better results than the other schemes. On the other hand, from Figure 6 we see that fairness seems to be unaffected by the average BER.

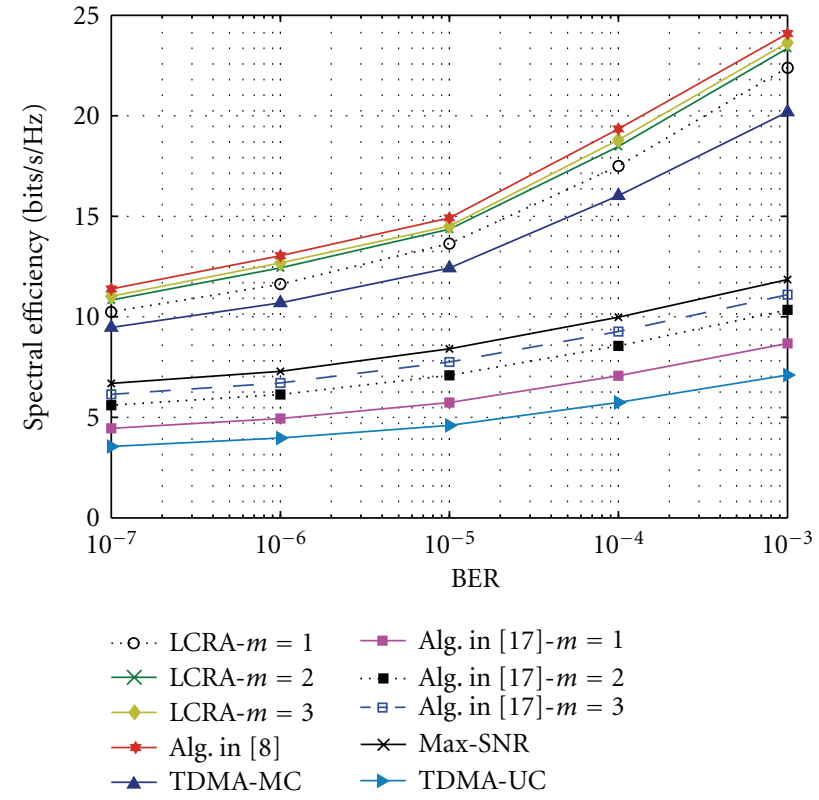

FIgURE 5: Spectral efficiency versus average BER.

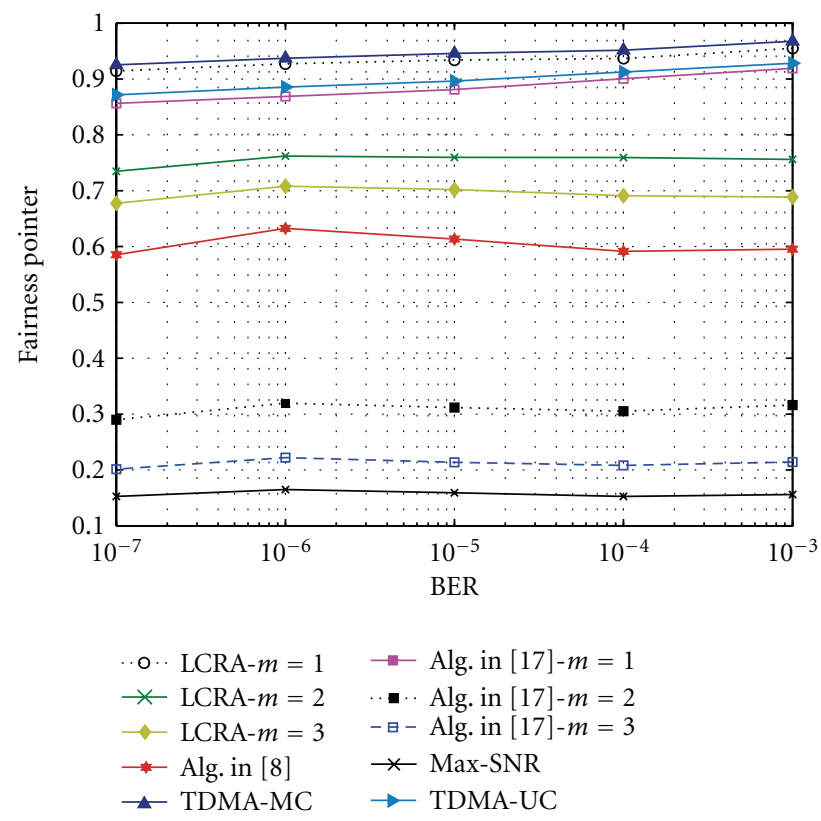

FIgURE 6: Fairness versus average BER.

6.3. LCRA Algorithm versus Multiple MGs. In this simulation case, various numbers of multicast groups are considered from 2 to 8 , whereby SNR $=20 \mathrm{~dB}$ and $\mathrm{BER}=10^{-6}$. Figure 7 depicts the spectral efficiency against the number of groups for all the aforementioned multicast schemes, while Figure 8 shows the fairness pointer against the number of MGs served by the BS.

From Figure 7, we can see the tradeoff between the guaranteed number of resources for each $\mathrm{MG}$ and the achieved system spectral efficiency and as the number of MGs increases, the system data rate increases too, because of 


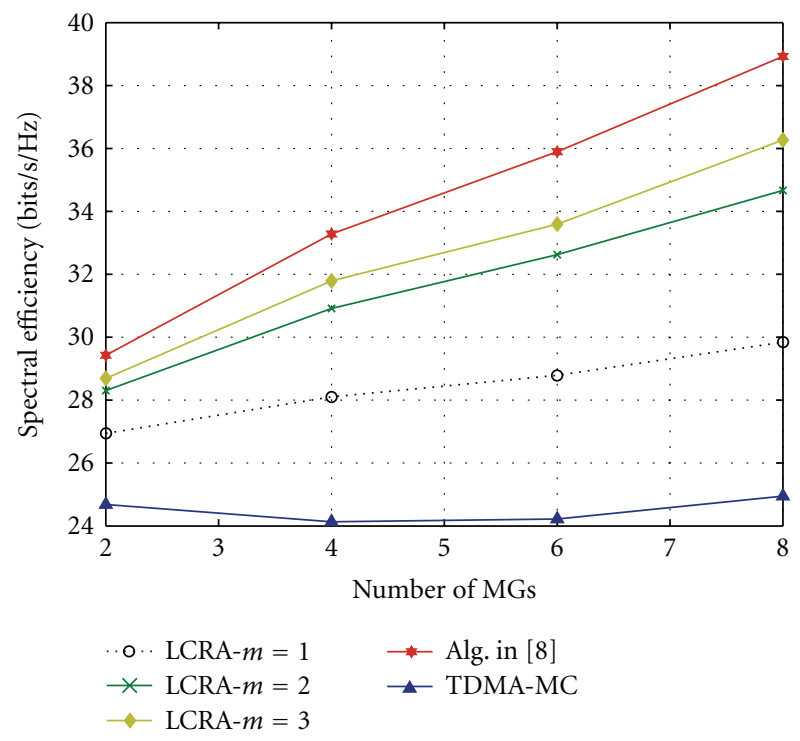

FIgURE 7: Spectral efficiency versus number of MGs.

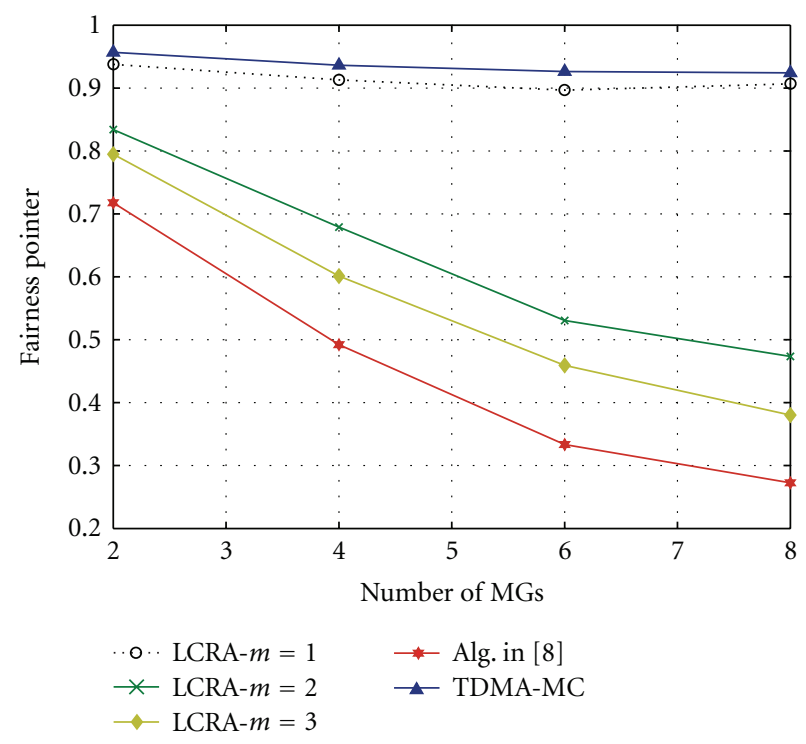

Figure 8: Fairness pointer versus number of MGs.

the additional multiuser diversity. In parallel with achieved spectral efficiency, the same happens with fairness pointer but in a different way. The difference is that fairness decreases as the number of MGs increases (Figure 8). According to conclusions in [31], even if the number of MGs tends to infinity, the performance of all schemes will reach a bound.

6.4. LCRA and Bandwidth Distribution. We consider two different multicast services which are provided to the available users. Thus, users are divided into two MGs, namely, MG1 and MG-2 and we also consider that we have $\left|\mathcal{K}_{1}\right|=$ $\left|\mathcal{K}_{2}\right|=8$ users in each group. Figures 9 and 10 plot the total spectral efficiency as well as the total fairness obtained

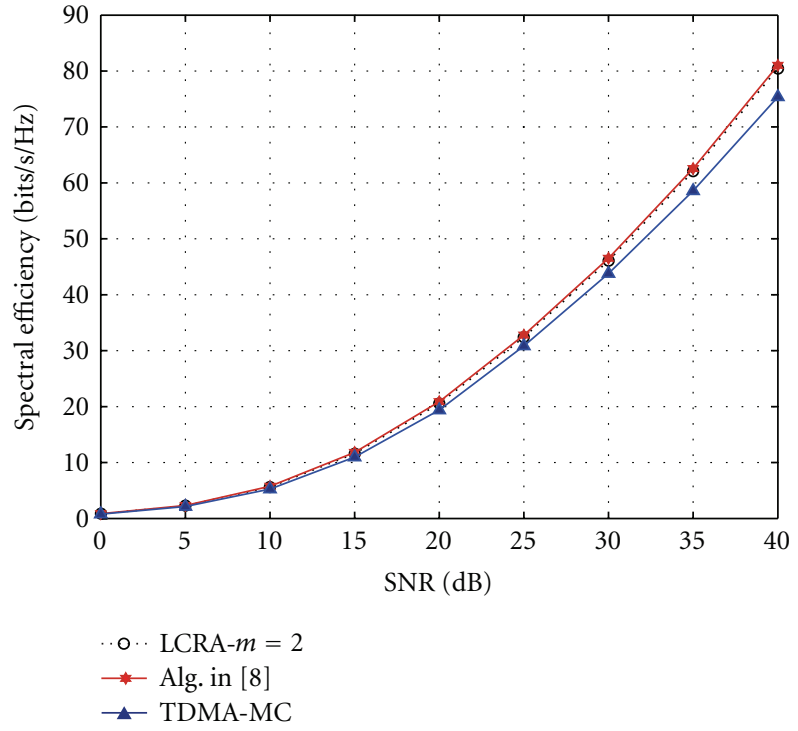

FIGURE 9: Spectral efficiency versus SNR-no pathloss difference between MGs.

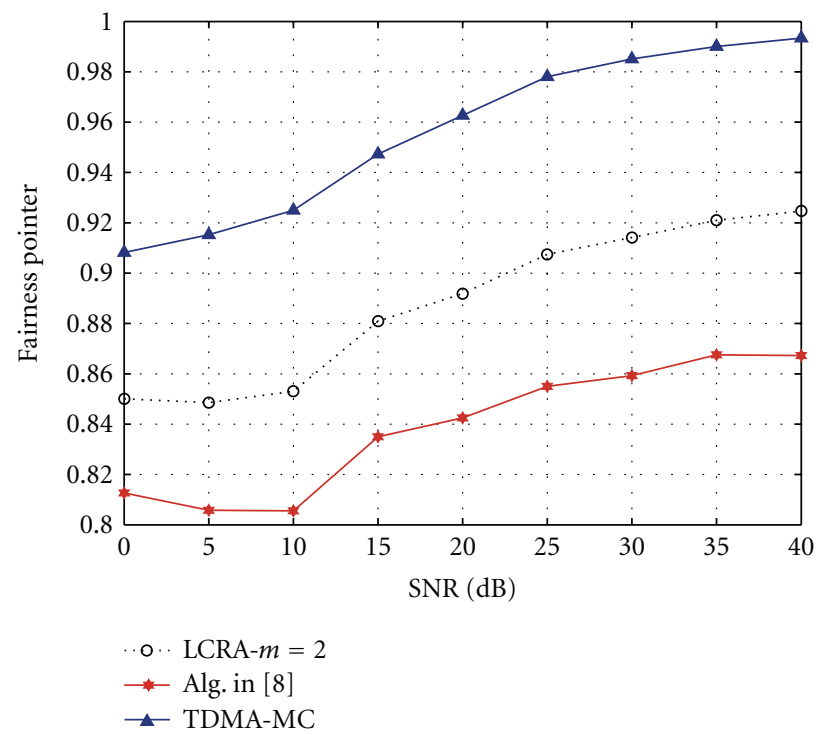

FIGURE 10: Fairness pointer versus SNR—no pathloss difference between MGs.

when there is no pathloss difference between the two MGs. Similar plots are shown in Figures 11 and 12 when there is $5 \mathrm{~dB}$ pathloss difference between the two MGs. Individual group transmission rates $(\mathrm{bits} / \mathrm{s} / \mathrm{Hz}$ ) when pathloss does not exist and when pathloss exists are given in Figures 13 and 14, respctively, while bandwidth distributions are shown in Figures 15 and 16 for the cases we study in this section. For ease of presentation, we consider 1 variant of the proposed LCRA algorithm with $m=2$ which means that, for each MG, we guarantee $C_{j}=\lfloor N /(2 \cdot 2)\rfloor=32$ subcarriers, where $j=1, \ldots, G$. 


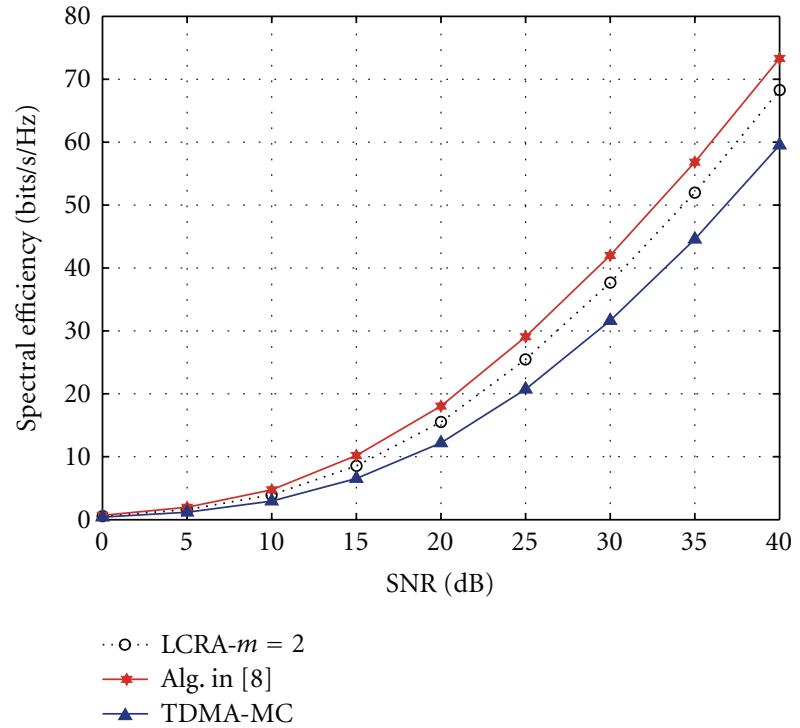

FIGURE 11: Spectral efficiency versus SNR—pathloss difference between MGs.

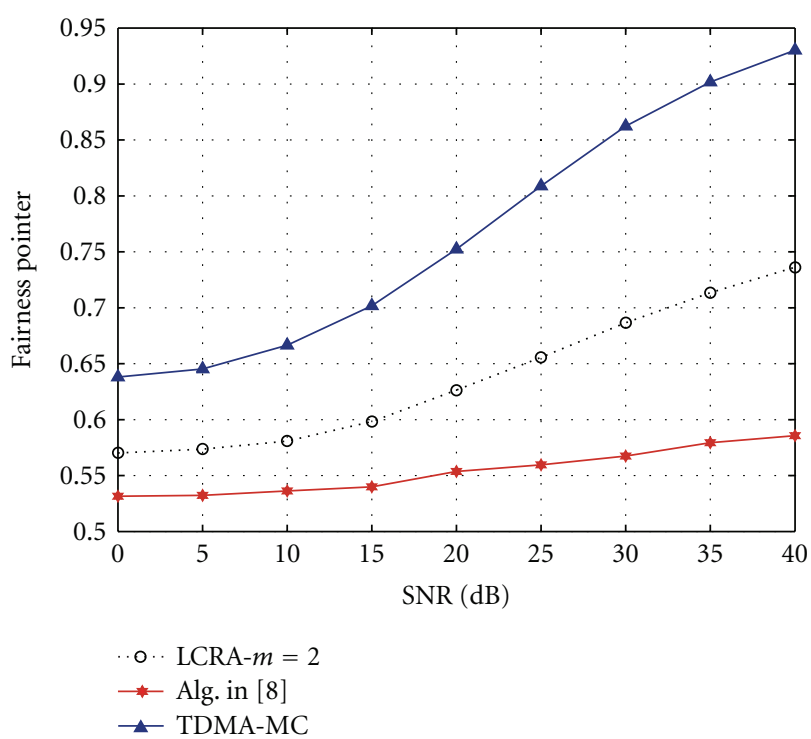

FIGURE 12: Fairness pointer versus SNR-pathloss difference between MGs.

From Figures 9 and 11, we see the negative impact of pathloss on the overall spectral efficiency as it is expected, for all schemes. Moreover, all schemes show similar performance in case there is no pathloss. On the other hand, when pathloss exists, LCRA gives lower system throughput from the algorithm in [8] and better results than TDMA (Figure 11). Figures 10 and 12 show the decrease in the fairness pointer for all schemes and the slight increase in fairness differences among all schemes (Figure 12) which pathloss brings about.

When there is no pathloss difference between the groups, individual MG rates seem to be very close as well as their

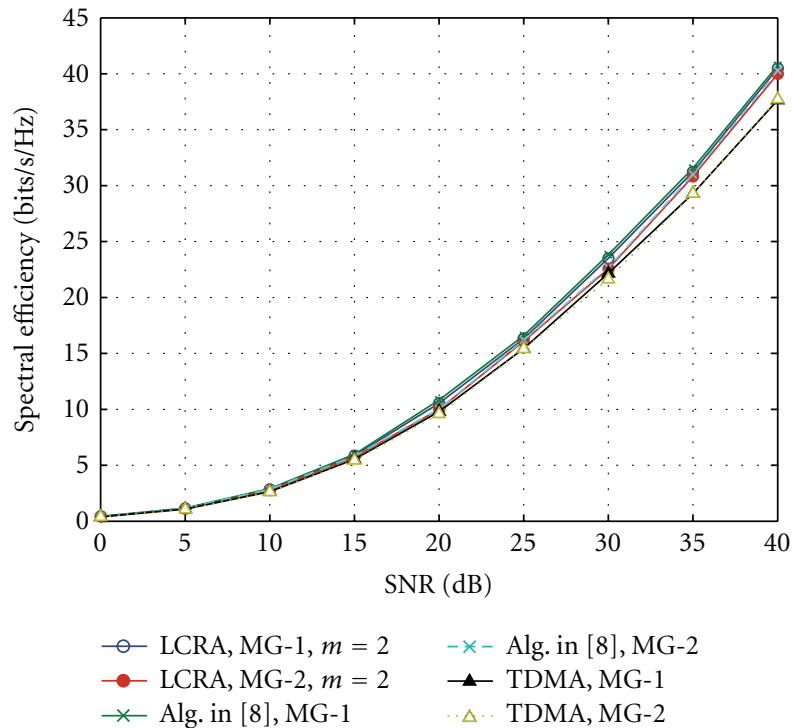

FIgure 13: Individual MG spectral efficiency versus SNR-no pathloss difference between MGs.

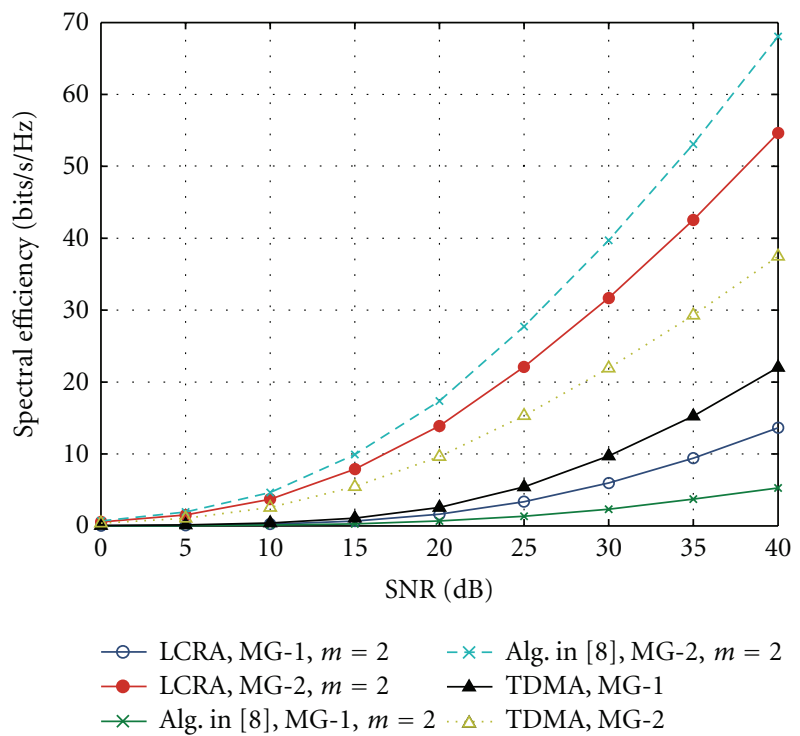

FIGURE 14: Individual MG spectral efficiency versus SNR—pathloss difference between MGs.

assigned spectrum. More specifically, the average channel distribution is 64.6 and 63.4 subcarriers for MG-1 and MG2 , respectively. The proposed LCRA gives 64.4 subcarriers to MG-1 and 63.6 to MG-2 on average, while with TDMA, strict fairness exists with 64 subcarriers to each group.

On the other hand, when pathloss is considered in simulations, we see that the group with less pathloss (MG-2) gives better performance than multicast group MG-1 in all schemes. The gap between the achievable bit rates of MG1 and MG-2 is wider with the scheme in [8] and smaller with TDMA than in LCRA as we see from plots in Figure 14. Moreover, Figure 16 shows that MG-1 in LCRA has 


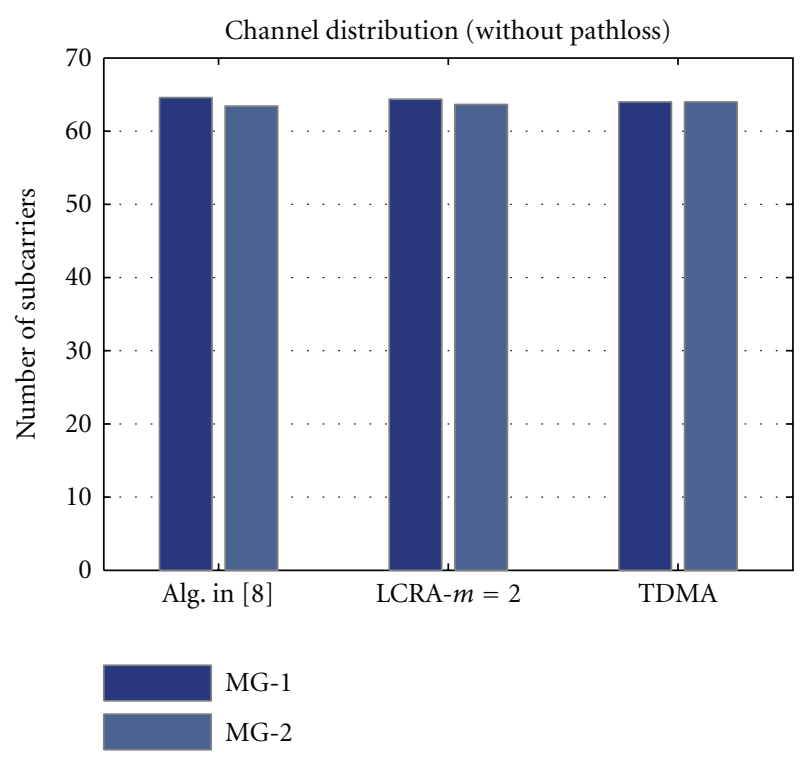

FIGURE 15: Channel distribution-no pathloss difference between MGs.

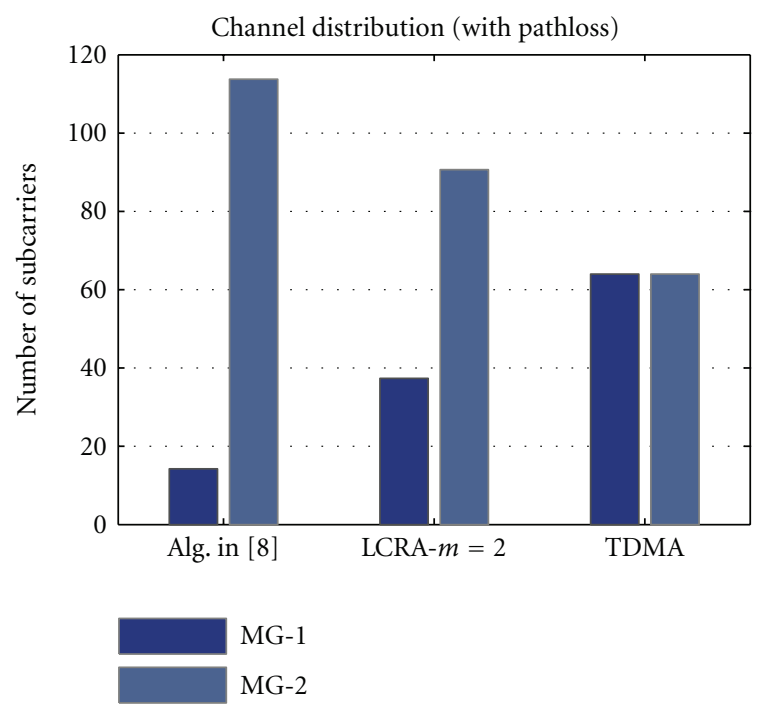

Figure 16: Channel distribution-pathloss difference between MGs.

a bigger share of bandwidth with 37.3 subcarriers on average than with the scheme in [8], which gives 14.3 subcarriers on average. In this case, when pathlosses exist, the proposed LCRA contributes more to system fairness. Note that, according to the parameter $m$, we can regulate system fairness as well as the bandwidth share of each multicast group.

\section{Conclusion}

In this paper, a resource allocation algorithm for the MIMO multicast systems over frequency-selective channels has been introduced. Multicasting enables multiple users to share a subcarrier and results have shown that this enhances significantly the total throughput. The capacity can become even higher by the presence of multiple MGs which bring more diversity into the system.

The proposed algorithm proved also to be very useful in systems wherein multiple MGs coexist, particularly in case their wireless link conditions are very different. LCRA is capable of providing bandwidth access guarantees to MGs in a flexible and controllable way that other reference schemes are unable to provide.

In parallel the proposed solution achieves low-complexity implementation by reducing the computational complexity from exponential to linear. Additionally, its computational complexity is independent of the presence of multiple antennas in both BS and users and as it is analyzed proved to be comparable with other low-complexity schemes.

\section{References}

[1] F. Hartung, U. Horn, J. Huschke, M. Kampmann, T. Lohmar, and M. Lundevall, "Delivery of broadcast services in $3 \mathrm{G}$ networks," IEEE Transactions on Broadcasting, vol. 53, no. 1, pp. 188-198, 2007.

[2] A. Alexiou, C. Bouras, V. Kokkinos, and E. Rekkas, "An improved mechanism for multiple MBMS sessions assignment in B3G cellular networks," Wireless Networks, vol. 16, no. 3, pp. 671-686, 2010.

[3] Y.-C. Lai, P. Lin, Y. Fang, and W.-H. Chen, "Channel allocation for UMTS multimedia broadcasting and multicasting," IEEE Transactions on Wireless Communications, vol. 7, no. 11, pp. 4375-4383, 2008.

[4] A. M. C. Correia, J. C. M. Silva, N. M. B. Souto, L. A. C. Silva, A. B. Boal, and A. B. Soares, "Multi-resolution broadcast/multicast systems for MBMS," IEEE Transactions on Broadcasting, vol. 53, no. 1, pp. 224-233, 2007.

[5] M. R. Chari, F. Ling, A. Mantravadi et al., "FLO physical layer: an overview," IEEE Transactions on Broadcasting, vol. 53, no. 1, pp. 145-159, 2007.

[6] J. Liu, W. Chen, Z. Cao, and K. B. Letaief, "Dynamic power and subcarrier allocation for OFDMA-based wireless multicast systems," in Proceedings of the IEEE International Conference on Communications (ICC '08), Beijing, China, May 2008.

[7] J. Y. Kim, T. Kwon, and D. H. Cho, "Resource allocation scheme for minimizing power consumption in OFDM multicast systems," IEEE Communications Letters, vol. 11, no. 6, pp. 486-488, 2007.

[8] J. Xu, S. J. Lee, W. S. Kang, and J. S. Seo, "Adaptive resource allocation for mimo-ofdm based wireless multicast systems," IEEE Transactions on Broadcasting, vol. 56, no. 1, pp. 98-102, 2010.

[9] I. G. Fraimis, V. D. Papoutsis, and S. A. Kotsopoulos, "A decentralized subchannel allocation scheme with Inter-cell Interference Coordination (ICIC) for multi-cell OFDMA systems," in Proceedings of the 53rd IEEE Global Communications Conference (GLOBECOM '10), December 2010.

[10] Y. Ben-Shimol, I. Kitroser, and Y. Dinitz, "Two-dimensional mapping for wireless OFDMA systems," IEEE Transactions on Broadcasting, vol. 52, no. 3, pp. 388-396, 2006.

[11] J. Jang and K. B. Lee, "Transmit power adaptation for multiuser OFDM systems," IEEE Journal on Selected Areas in Communications, vol. 21, no. 2, pp. 171-178, 2003.

[12] G. Li and H. Liu, "On the optimality of the OFDMA network," IEEE Communications Letters, vol. 9, no. 5, pp. 438-440, 2005. 
[13] T. C. H. Alen, A. S. Madhukumar, and F. Chin, "Capacity enhancement of a multi-user OFDM system using dynamic frequency allocation," IEEE Transactions on Broadcasting, vol. 49, no. 4, pp. 344-353, 2003.

[14] V. D. Papoutsis, I. G. Fraimis, and S. A. Kotsopoulos, "Fairness-aware resource allocation for the SISO downlink over frequency-selective channels," in Proceedings of the IEEE Wireless Communications and Networking Conference (WCNC'10), April 2010.

[15] M. Ergen, S. Coleri, and P. Varaiya, "Qos aware adaptive eesource allocation techniques for fair scheduling in OFDMA based broadband wireless access systems," IEEE Transactions on Broadcasting, vol. 49, no. 4, pp. 362-370, 2003.

[16] C. S. Bae and D. H. Cho, "Fairness-aware adaptive resource allocation scheme in multihop OFDMA systems," IEEE Communications Letters, vol. 11, no. 2, pp. 134-136, 2007.

[17] V. D. Papoutsis, I. G. Fraimis, and S. A. Kotsopoulos, "Resource allocation algorithm for MIMO-OFDMA systems with minimum resources guarantee," in Proceedings of the IEEE International Conference on Electronics, Cicuits and Systems (ICECS '10), Athens, Greece, December 2010.

[18] P. W. C. Chan and R. S. Cheng, "Capacity maximization for zero-forcing MIMO-OFDMA downlink systems with multiuser diversity," IEEE Transactions on Wireless Communications, vol. 6, no. 5, pp. 1880-1889, 2007.

[19] G. Li and H. Liu, "On the optimality of downlink OFDMA MIMO systems," in Proceedings of the 38th IEEE Asilomar Conference on Signals, Systems and Computers, pp. 324-328, California, Calif, USA, November 2004.

[20] Z. J. Guan, H. Li, C. Q. Xu, X. L. Zhou, and W. J. Zhang, "Adaptive subcarrier allocation for MIMO-OFDMA wireless systems using Hungarian method," Journal of Shanghai University, vol. 13, no. 2, pp. 146-149, 2009.

[21] J. Xu, J. Kim, W. Paik, and J. S. Seo, "Adaptive resource allocation algorithm with fairness for MIMO-OFDMA system," in Proceedings of the IEEE Vehicle Technology Conference (VTC '06), Melbourne, Australia, May 2006.

[22] A. N. Zaki and A. O. Fapojuwo, "A graph-based resource allocation algorithm for downlink MIMO-OFDMA networks," in Proceedings of the IEEE Global Telecommunications Conference (GLOBECOM '09), December 2009.

[23] M. S. Maw and I. Sasase, "Resource allocation scheme in MIMO-OFDMA system for user's different data throughput requirements," IEICE Transactions on Communications, vol. E91-B, no. 2, pp. 494-504, 2008.

[24] B. Da and C. C. Ko, "Resource allocation in downlink MIMOOFDMA with proportional fairness," Journal of Communications, vol. 4, no. 1, pp. 8-13, 2009.

[25] S. Xiao, X. Xiao, B. Li, and Z. Hu, "Adaptive subcarrier allocation for multiuser MIMO OFDM systems in frequency selective fading channel," in Proceedings of the IEEE International Wireless Communications, Networking \& Mobile Computing Conference, Wuhan, China, 2005.

[26] X. Lu and Z. Li, "An adaptive resource allocation algorithm based on spatial subchannel in multiuser MIMO/OFDM systems," in Proceedings of the IEEE International Conference on Communications (ICC '08), J. Cai and X. Chen, Eds., Beijing, China, May 2008.

[27] H. Rohling and R. Grunheid, "Performance comparison of different multiple access schemes for the downlink of an OFDM communication system," in Proceedings of the IEEE Vehicle Technology Conference (VTC'97), May 1997.
[28] S. T. Chung and A. J. Goldsmith, "Degrees of freedom in adaptive modulation: a unified view," IEEE Transactions on Communications, vol. 49, no. 9, pp. 1561-1571, 2001.

[29] G. Li and H. Liu, "Downlink radio resource allocation for multi-cell OFDMA system," IEEE Transactions on Wireless Communications, vol. 5, no. 12, pp. 3451-3459, 2006.

[30] Z. Shen, J. G. Andrews, and B. L. Evans, "Adaptive resource allocation in multiuser OFDM systems with proportional rate constraints," IEEE Transactions on Wireless Communications, vol. 4, no. 6, pp. 2726-2737, 2005.

[31] C. Suh and J. Mo, "Resource allocation for multicast services in multicarrier wireless communications," IEEE Transactions on Wireless Communications, vol. 7, no. 1, pp. 27-31, 2008. 

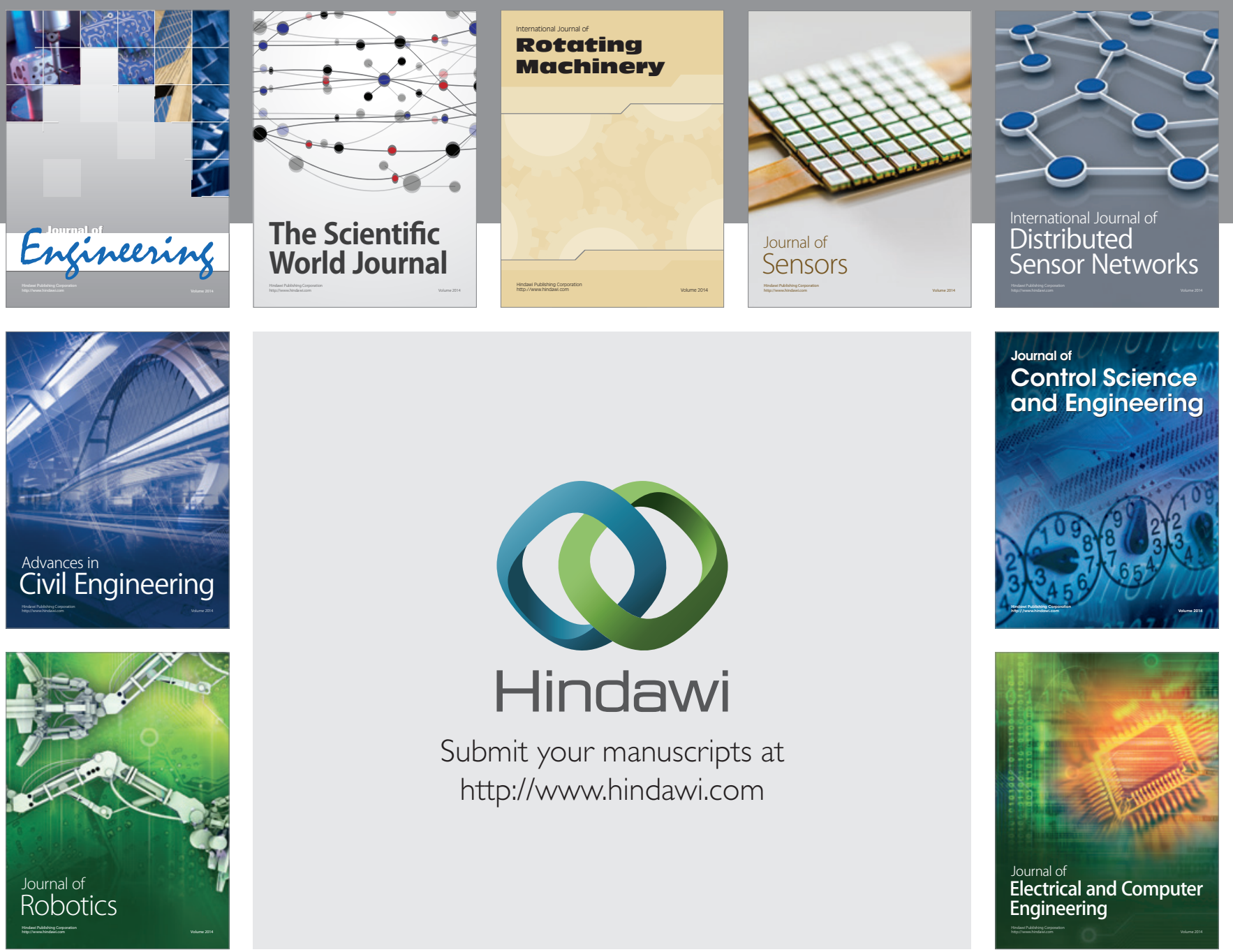

Submit your manuscripts at

http://www.hindawi.com
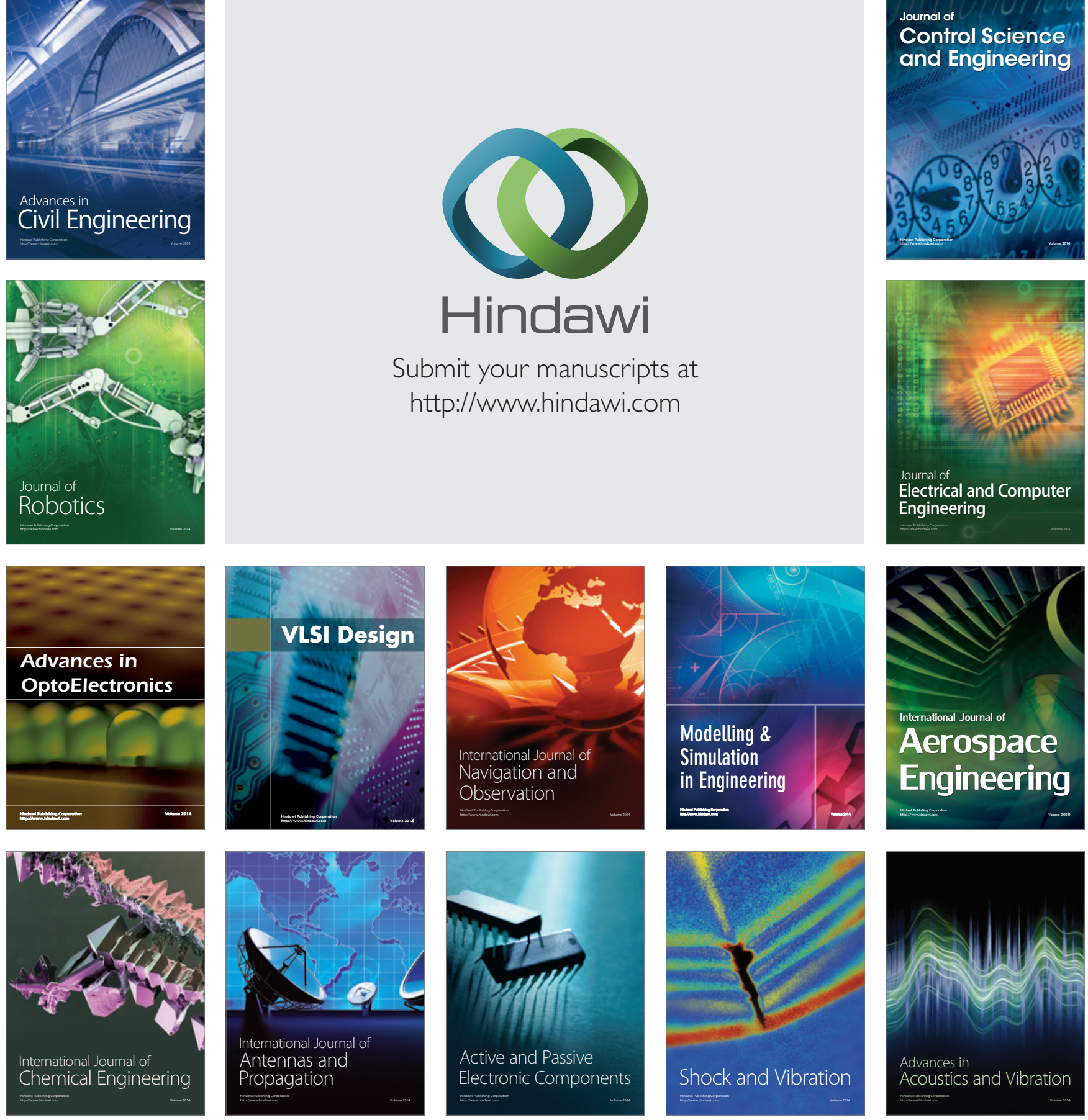